\title{
Economía campesina y territorio en las políticas de desarrollo rural ${ }^{*}$
}

\author{
Wilson González Santos ${ }^{1}$ \\ Universidad Pedagógica y Tecnológica de Colombia (Colombia)
}

Recibido: diciembre 10 de 2014 - Revisado: marzo 20 de 2015 - Aceptado: agosto 5 de 2015

Referencia norma APA: González-Santos, W. (2015). Economía campesina y territorio en las políticas de desarrollo rural. Revista Científica Guillermo de Ockham, 13(2), 101-106.

\section{Resumen}

Las nuevas concepciones del desarrollo rural intentan aproximarse a su dinámica multidimensional. Consecuentemente, la lógica campesina requiere un análisis especial que se acerque a su compleja cotidianidad. No obstante, la particularidad de su territorio excluye la interpretación reduccionista de la cartografía euclidiana, propia de la política agraria colombiana. De acuerdo con lo anterior, en el sector rural un espacio concebido por los tomadores de decisión como un objeto diferenciado por limites físicos, políticos o administrativos se convierte en el territorio que se debe intervenir y que desconoce la construcción social y por ende, los procesos de articulacion que le han dado su significado. Los campesinos de Latinoamérica han forjado y construido un territorio que trasciende esta concepción estatal y por lo tanto es disímil a las contenidas en las políticas de intervención. El presente trabajo cuestiona la pertinencia de los instrumentos políticos para comprender la complejidad del mundo campesino latinoamericano, traducidos en la territorialidad institucional.

Palabras clave: Espacio vivido, campesino, lógica, complejidad

\section{Rural economy and territory in the rural development policies}

\section{Abstract}

The new conceptions for rural development to try to approach multiple dimensions dynamics. As a result, the peasant logic requires a special analysis approaches its complex daily. However, the particularity of its territory excludes the reductive interpretation of Euclidean cartography, typical of Colombian agricultural policy. Due to the above, in the rural sector with a designed space - from policy makers, as a distinct object by physical, political or administrative limits becomes the territory to be intervener and ignores the social construction and thus, the joining processes that have given it its meaning. The peasants of Latin America have made and built a territory that transcends this state conception and which is different from the intervention policies. This paper doubts the relevance of policy instruments to understand the complexity of Latin American peasant world, as such institutional territoriality.

Keywords: Lived space, farmer, logic, complexity

* El presente artículo es producto del proyecto de investigación doctoral Dinámica campesina y estructura agraria: el sistema agroalimentario de la papa en el municipio de Villapinzón. Programa de Doctorado en geografía. Convenio UPTC-IGAC del año 2014.

1. Ingeniero agrónomo. Magíster en Desarrollo Rural. Doctorando en geografía Convenio UPTC-IGAC. Grupo de Investigación Competitividad y Desarrollo Local (Codel). E-mail: wilson.gonzalez@uptc.edu.co 


\section{Economia rural e do território nas políticas de desenvolvimento rural}

\section{Resumo}

As novas concepçóes do Desenvolvimento Rural tentam aproximar sua dinâmica de múltiplas dimensóes. Como resultado, a lógica do camponês precisa de uma análise especial de abordar o seu atividade todos os dia. No entanto, a particularida de de seu território não considera a interpretação redutora da cartografia euclidiana, típica da política agrícola colombiana. Por isso nas áreas rurais um espaço concebido pelos decisores politicos como um objecto caracterizado por limites físicos, politicos e adminstrativos torna-se o território intervenção e ignora a construção social e, portanto, os processos comuns que deram significado. Os camponeses da América Latina ter forjado e construído um território que transcende essa concepção do Estado e, portanto, é diferente para aqueles em políticas de intervenção. Este artigo coloca em dúvida a relevância dos instrumentos políticos para compreender a complexidade do mundo camponês latino-americana, como tal territorialidade institucional.

Palavras-chave: Espaço de vida, camponês, lógica, complexidade

\section{Introducción}

La permanente dinámica del contexto rural, traducida en nuevas características que lo definen, ha motivado el surgimiento de concepciones que trascienden su lectura como un espacio proveedor de bienes y servicios meramente agropecuarios. En este sentido, existen al menos dos condicionantes que originan estas concepciones: el desgate del modelo de la revolución verde y las interpretaciones teóricas relacionadas con la integralidad del espacio rural (González, 2009). Con referencia a este último elemento, es importante indicar que una definición holística de lo rural exige una aproximación no sujeta a apuestas propias de un determinado ámbito disciplinar que no solo distan de su complejidad, sino que también motivan intervenciones equivocas al no corresponder con su quehacer y por ende, su problemática. Si el territorio se manifiesta como una forma objetizada y consciente del espacio (López, 2015) -como consideremos dicho espacio-, así también ejerceremos territorialidad sobre él. De esta manera, el uso de la territorialidad en un espacio lleva de manera inherente las herramientas usadas para su comprensión.

Con la revisión de la nueva ruralidad como apuesta conceptual para comprender la complejidad rural, el autor subraya la necesidad de admitir concepciones que trasciendan la mirada del territorio campesino como un espacio objetivo representado por una cartografía euclidiana y admitan la inclusión de imaginarios y subjetividades que históricamente le han dado su impronta. El texto finaliza con una crítica a la política pública agraria que no considera la especificidad del territorio campesino y lo aborda desde perspectivas ligadas al reduccionismo y a la objetividad positivista.

\section{El desarrollo rural contemporáneo}

El espacio rural ha sido objeto de distintos acercamientos, entre los cuales destacan las posturas técnica, política y utópica, entendidas como estrategias de intervención "adecuadas" para concebirlo y, en función de ello, proponer caminos de desarrollo. No obstante, distan de la mirada integral necesaria para su comprensión.

En particular para Latinoamérica, ha emergido la nueva ruralidad como una corriente teórica que intenta comprender los cambios ocurridos en el espacio rural (Llambi, 2004). De acuerdo con Pérez et al., (2000), "el desarrollo sectorial agrícola y pecuario está determinado en forma creciente por aspectos cuyo origen son procesos económicos, culturales, sociales, culturales y políticos que rebasan su mero carácter de sector económico-productivo" (p. 16).

Según Gómez (2001), un perfil de la nueva ruralidad puede construirse a partir de una comparación entre las características que tipifican un escenario tradicional (vieja ruralidad) y las que particularizan la nueva ruralidad (ver Tabla 1). En este sentido la nueva ruralidad es entendida como un espacio cuya dinámica propicia un acercamiento y en ningún momento desconoce las crisis del sector agrario en los últimos años.

Como puede inferirse, la tipología destaca nuevos condicionantes para el sector rural que obligan a detenerse en sus relaciones con lo urbano y, consecuentemente, propone una concepción de territorio que trasciende los límites políticos e institucionales al rebasar las fronteras que lo señalaban como un espacio meramente agropecuario. Si se acepta esta manera de abordar el territorio, es 
Tabla 1

El espacio rural a partir de dos interpretaciones

\begin{tabular}{lll}
\hline \multicolumn{1}{c}{ Ítem } & Vieja ruralidad & \multicolumn{1}{c}{ Nueva ruralidad } \\
\hline Oferta & Agropecuaria & $\begin{array}{l}\text { Agropecuaria, am- } \\
\text { biental, recreativa y } \\
\text { servicios, entre otras }\end{array}$ \\
\hline Lugar en el desarrollo & Residual & Principal \\
\hline $\begin{array}{l}\text { Infraestructura } \\
\text { y servicios }\end{array}$ & Menor & $\begin{array}{l}\text { Puede ser equitativo } \\
\text { con lo urbano e incluso } \\
\text { mayor }\end{array}$ \\
\hline $\begin{array}{l}\text { Naturaleza del } \\
\text { empleo }\end{array}$ & Agropecuario & $\begin{array}{l}\text { Toma cada vez mayor } \\
\text { importancia el empleo } \\
\text { no agrícola (ENA) }\end{array}$ \\
\hline $\begin{array}{l}\text { Bienestar de la } \\
\text { población }\end{array}$ & Menor & $\begin{array}{l}\text { Puede ser equitativo } \\
\text { con lo urbano e incluso } \\
\text { mayor }\end{array}$ \\
\hline
\end{tabular}

Fuente: Elaboración propia a partir de Gómez (2001).

necesario sobrepasar los enfoques sectoriales entre los dos ámbitos (rural y urbano) (Schejtman \& Berdegué, 2004) y acercarse al estudio de las relaciones que los articulan. González (2009), indica que en dicha articulación se pueden generar al menos tres escenarios:

- Cambios dentro de los dos espacios.

- Mantenimiento de las características particulares de cada uno.

- Surgimiento de un nuevo componente producto de dicha relación.

Los dos últimos son particularmente interesantes para abordar en el siguiente aparte, la lógica de la economía campesina.

\section{La lógica campesina}

Los discursos anticampesinistas que pregonaban la idea de la desaparición de la población por el simple hecho de su aparente ineficiencia económico-productiva, resultan poco pertinentes si se tiene en cuenta que la realidad de la población rural latinoamericana muestra una "inusual" fortaleza o "adaptabilidad" para mantenerse.

Si se acepta que a la luz de los indicadores económicos no es posible explicar la permanencia de este grupo humano, cabe inferir que la persistencia campesina obliga a ser analizada desde una perspectiva particular ajena a las explicaciones que propondría la tradicional sociología rural (léase en el apartado anterior "vieja ruralidad") que enfatiza las distinciones entre lo rural y lo urbano (Hillyard, 2007).

Núñez y Díaz (2006) proponen las siguientes componentes para caracterizar la economía campesina:

- El autoconsumo en alto grado, con el predominio de los valores de uso sobre los bienes mercantiles.

- El trabajo familiar y el uso de energía humana y animal predominan sobre el pago jornales externos y la energía mecánica.

- La reproducción familiar, como directriz de decisión de la existencia.

- La producción diversificada sin el condicionamiento de las economías de escala.

De vuelta a Núñez y Díaz (2006), la comunidad campesina se considera un modo especial creado por la humanidad para relacionarse con la naturaleza y extraerle los bienes necesarios para su subsistencia. De acuerdo con lo anterior, su lógica dista de la del moderno sector agroindustrial, dada su particular racionalidad: un empresario tiene como fin maximizar su ingreso neto para lograr una mayor rentabilidad y un campesino potenciar su renta agrícola familiar para mantener su supervivencia. ${ }^{2}$

Anteriormente se mencionaron los posibles contextos resultantes de la articulación urbana rural. Ahora bien, es posible ejemplificar estos eventos con una mirada a la racionalidad de la comunidad campesina.

El mantenimiento de las características del ámbito rural hace relación a las denominadas acciones afectivas en las que los campesinos persisten a pesar de su aparente ineficiencia económica. Esta cuestión es comprensible en el terreno de la racionalidad campesina, definida como un conjunto de reglas o principios que le dan sentido a la acción (Landini, 2011). Si se acepta que para este grupo humano la supervivencia de la familia no solo es la bitácora para su actuar, sino muy especialmente el soporte de su persistencia, es posible entender la existencia de prácticas ancestrales distantes a la lógica de la eficiencia económica: una actividad agrícola es valorada no solo por su producción y venta comercial, sino principalmente por la posibilidad de vincular a los miembros de la familia campesina y producir un importante excedente para su autoconsumo: el cultivo del maíz tradicional en el altiplano cundiboyacense ejemplifica esta situación.

2. La renta agrícola familiar o ingreso disponible se define como la diferencia entre el valor de la producción (venta más autoconsumo) y el pago a factores externos a la unidad productiva. 
La adaptabilidad al contexto globalizante mediada tanto por la economía moral (derivada de la acción afectiva) como por la racionalidad económica, ilustra el surgimiento de un nuevo componente. La periurbanización, como proceso que evidencia tanto la difusión urbana como la transformación espacial en lo rural (Hernández, Martínez \& Méndez, 2014), supone espacios en los cuales se amalgaman intensamente los elementos rurales y urbanos.

Las anteriores consideraciones sostienen el capital social "entendido como el contenido de ciertas relaciones y estructuras sociales; es decir, las actitudes de confianza que se dan en combinación con conductas de reciprocidad y cooperación" (Durston, 2002, p. 15), como fundamento que posibilita en los actores los procesos de articulación entre lo rural y lo urbano. Es decir, las relaciones sociales en un espacio determinado configuran el territorio como una construcción social.

Por consiguiente, acercarse a la lógica campesina implica considerar una mezcla de factores que explican su accionar: su persistencia trasciende la mera mercantilización de la producción.

\section{Territorio y territorialidad en el sector campesino}

Lo anteriormente expuesto permite afirmar que el territorio campesino contiene un acento tanto objetivo como subjetivo que lo distingue, y al ser una construcción social se encuentra sujeto a una dinámica que depende de los procesos de articulación de quienes en él participan.

El territorio, además de ser un espacio que se ha valorizado instrumentalmente (bajo el aspecto ecológico, económico o geopolítico), también lo es culturalmente. En el proceso de valorización, la interacción social entre los habitantes permite que el territorio sea significado, transformado y vivido. En el territorio se inscriben las características de quienes lo habitan, razón por la cual es también fuente de recuerdos (Hernández, 2011).

De igual forma, la comprensión del territorio rural nos sitúa en un escenario en el que se hace necesario trascender las interpretaciones cartesianas del espacio propias de la geografía cuantitativa y su modelo positivista (Delgado, 2003), para aproximarnos a enfoques que admitan no solo el estudio de eventos complejos, multidimensionales y no susceptibles de ser reducidos por la metodología científica, sino también propios de una lógica distinta. La persistencia de las acciones afectivas de los campesinos, ejemplifican esta situación.
Lefebvre (1974), en la trialéctica de la espacialidad, propone la confluencia de la práctica espacial (espacio percibido), de las representaciones del espacio (concebido) y de los espacios de representación (vivido). Por su lado, Franco (2004) entiende la espacialidad como un componente de las relaciones sociales y no como un reflejo de estas.

En el Tabla 2 se elaboran los eventos de la economía campesina que corresponderían a los espacios propuestos por la trialéctica espacial. Particularmente, los espacios concebidos y vividos son difícilmente comprendidos a partir de las herramientas cuantitativas geográficas (léase cartográficas euclidianas).

Tabla 2

La trialéctica espacial y la economía campesina

\begin{tabular}{cl}
\hline \multicolumn{1}{c}{ Espacio } & \multicolumn{1}{c}{ Ejemplos de eventos } \\
\hline Espacio percibido & $\begin{array}{l}\text { Minifundio, zonas con predominio de altas } \\
\text { pendientes, alta diversificación, etc. }\end{array}$ \\
\hline Espacio concebido & $\begin{array}{l}\text { Alto compromiso con la supervivencia y persis- } \\
\text { tencia de la unidad familiar. }\end{array}$ \\
\hline Espacio vivido & $\begin{array}{l}\text { Múltiples lógicas: con distinto arreglo al tránsi- } \\
\text { to capitalista. }\end{array}$ \\
\hline
\end{tabular}

Fuente: Elaboración propia con base en Urrejola (2005).

En el espacio concebido y vivido se introduce, a partir de la metodología científica, el análisis de lógicas aparentemente difusas. Como se había indicado anteriormente, estas lógicas escapan a la mirada "restringida" de las valoraciones cuantitativas propias -entre otras cosas- de imaginarios individuales y colectivos que históricamente han reafirmado este territorio y que no percibidos materialmente poseen una alta significación.

Mientras la cartografía cartesiana o institucional se encarga preferentemente de la representación del espacio percibido, la cartografía social o participativa trabaja con los otros dos (Barrera, 2009). La cartografía social vincula en su construcción a quienes habitan y "construyen" el espacio a que le dan un significado: el territorio.

Particularmente, la intervención en el territorio rural se ha permeado con herramientas que posibilitan la cartografía social. Es importante destacar la planeación y el diagnóstico rural participativo (DRP), en el cual la comunidad hace una radiografía de su situación con el uso de herramientas de investigación accesibles a su comprensión y caracterizadas por su alto contenido simbólico, basados en sus propios conceptos y criterios de explicación (Expósito, 2003).

No obstante, la conveniencia de la cartografía social como instrumento de comprensión de la economía 
campesina, se debate en una atmósfera ajena a las preocupaciones del espacio social rural que se elabora y reelabora permanentemente. Los procesos de articulación rural urbanos cada vez más intensos, requieren miradas no solo interdisciplinares (entre estos dos ámbitos), sino muy especialmente transdisciplinares que reconozcan la yuxtaposición funcional de estos dos sectores.

\section{A manera de conclusión: transitar un arduo camino: política agraria al debate}

La política pública colombiana para el sector agrario promueve su inserción en el comercio internacional, con actividades agrícolas y pecuarias en función no de las ventajas comparativas dadas por las condiciones edafoclimáticas, sino especialmente de las competitivas relacionadas con las externalidades positivas (Espinal \& Martínez, 2006), tales como el desarrollo de las cadenas agroexportadoras de cultivos permanentes e identifica los denominados productos promisorios en función de su comportamiento en los tratados comerciales (El Tiempo, 2006). La hipótesis se basa en que el flujo y el balance comercial son la bitácora para el crecimiento y desarrollo (Tobasura, 2009). Con esta lectura, el campesino debe hacer su conversión a empresario para usufructuar los beneficios de dicha apuesta. Sin embargo, ¿la lógica campesina opera con este modelo?

Un colectivo cuya complejidad se corresponde con múltiples escenarios y, en consecuencia, con múltiples racionalidades ${ }^{3}$ inherentes a su objetivo de permanencia y perdurabilidad, no se puede comprender con una óptica reduccionista ligada a una apuesta que se soporta en criterios estrictamente economicistas.

Más aún, el soporte conceptual de las cadenas con ventajas comparativas y competitivas, se deriva de indicadores propios del "lenguaje científico positivista" representado en la cartografía institucional, en la cual las relaciones sociales no adquieren importancia y distan de la comprensión holística -pero también compleja- del sector campesino, cuya teoría de la acción social armoniza elementos racionales e irracionales (Tobasura, 1996).

¿No es la territorialidad usar el poder a fin de excluir las micropolíticas posibles que pueden surgir luego de un proceso de cartografía social? El trabajo continúa: repensar la neutralidad de la ciencia y su utilidad como soporte para la operatividad de una política pública en el territorio campesino, es una imperiosa necesidad.

\section{Referencias}

Barrera, S. (2009). Reflexiones sobre sistemas de información geográfica Participativos (SIGP) y cartografía social. Cuadernos de geografía. Revista Colombiana de geografía. (18), pp. 9-23.

Delgado, O. (2003). Debates sobre el espacio en la geografía contemporánea. Universidad Nacional de Colombia. Unibiblos. Bogotá. $253 \mathrm{p}$

El Tiempo. (14 de julio de 2006). El Ministerio de Agricultura lanzó la llamada Apuesta Exportadora Agropecuaria [Noticia en línea]. Recuperado de http://goo.gl/vs6CWY

Durston, J. (2002). El capital social campesino en la gestión del desarrollo rural. Santiago de Chile: CEPAL.

Expósito, M. (2003). Diagnóstico rural participativo. Una guía práctica. Santo Domingo: Centro Cultural Poveda.

Espinal, C., \& Martínez, H. (2005). La competitividad de las cadenas agroproductivas en Colombia. Bogotá: IICA.

Franco, D. (2004). Espacios de representación e imaginario social en aldeas escolares de la provincia del Chubut. Párrafos geográficos. 2(2). Recuperado de http://goo.gl/0wntCM

Gómez, S. (2001). ¿Nueva ruralidad? Un aporte al debate. Revista Sociedade e Agricultura. 17, 1-5.

González, W. (2009). Educación y entorno en la ingeniería agronómica. Tunja: Universidad Pedagógica y Tecnológica de Colombia.

Hernández, L. (2011). Geografía de los territorios rurales. Notas para su análisis. Revista Catalana de Geografía. 16(42). Recuperado de http://www.rcg.cat/articles.php?id=203

Hernández, J., Martínez, B., \& Méndez, J. (2014). Reconfiguración territorial y estrategias de reproducción social en el periurbano poblano. Cuadernos de Desarrollo Rural. 11(74), 13-34.

Hillyard, S. (2007). Sociology of Rural Life. Oxford: Berg Publishers.

Landini, F. (2011). Racionalidad económica campesina. Mundo Agrario. 2(23), 9-23.

Lefebvre, H. (1974). La production de l'espace social. Anthropos: Barcelona.

Llambi, L. (2004). Nueva ruralidad, multifuncionalidad de los espacios rurales y desarrollo local endógeno. En E. Pérez \& M. Farah (Com.), Desarrollo Rural y Nueva Ruralidad en

3. En términos weberianos: en las economías campesinas “todo cálculo natural está orientado según su esencia más íntima en el consumo: Cobertura de necesidades" (Weber, 1942). 
América Latina y la Unión Europea (pp. 81-100). Bogotá: Pontificia Universidad Javeriana.

López, L. (Dir.). (2015). Diccionario de geografía aplicada y profesional. España: Universidad de León.

Núñez, I., \& Díaz, M. (2006). Innovación en la comunidad y economía campesina. Trabajo presentado en I Congreso Iberoamericano de Ciencia, Tecnología, Sociedad e Innovación, México.

Pérez, E., Farah, M., \& Rojas, M. (2000). Reconstruir la confianza en Colombia: nueva institucionalidad en el sector rural. Bogotá: Pontificia Universidad Javeriana.

Schejtman, A., \& Berdegué, J. (2004). Desarrollo territorial rural. Santiago de Chile: IDA-BID.

Soja, E. (1980). The Socio-Spatial Dialectic. Annals of the Association of American Geographers, 70(2), 207-225.
Tobasura, I. (2009). De campesinos a empresarios: la retórica neoliberal de la política agraria en Colombia. Cuaderno Venezolano de Sociología, 17(1), 21-34.

Tobasura, I. (1996). Racionalidad y procesos de racionalización en sociedades campesinas. Cuadernos de Desarrollo Rural. Pontificia Universidad Javeriana. 36 (1). pp. 45-55.

Urrejola, L. (2005). Hacia un concepto de espacio en antropología. Algunas consideraciones teórico-metodológicas para abordar su análisis (tesis de grado inédita). Universidad de Chile, Chile.

Universidad del Valle de Guatemala. (2010). Diagnostico Rural participativo. Centro de Desarrollo Rural. Recuperado de http://goo.gl/wLyYl5.

Weber, M. (1942). Historia económica general. México: Fondo de Cultura Económica. 